\title{
Development and Evaluation of Acyclovir Loaded Poly Lactic-Co-Glycolic Acid Nanoparticles for Ocular Drug Delivery
}

\author{
Harshal Dilip Mahajan ${ }^{1, *}$, Rajendra Dayaram Wagh', Dheeraj Tukaram Baviskar ${ }^{2}$ \\ 'Dhule Charitable Society's Annasaheb Ramesh Ajmera College of Pharmacy, Nagaon, Dhule, Maharashtra, INDIA. \\ 2Dr. Uttamrao Mahajan College of B. Pharmacy, Chalisgaon, Jalgaon, Maharashtra, INDIA.
}

\begin{abstract}
Objectives: The objective of present study aim towards promising potential of acyclovir containing Poly Lactic-Co-Glycolic Acid based nanoparticles for delivering drug to ocular cavity and could be view as alternative to conventional dosage form. Methods: The acyclovir containing Poly LacticCo-Glycolic Acid based polymeric nanoparticles were prepared by using nanoprecipitation technique and the optimized formulation was evaluated for particle size, encapsulation efficiency, in vitro release study, Differential Scanning Calorimetry, X-Ray Diffraction, Scanning Electron Microscopy, redispersibility, swelling index, ex vivo study and stability studies. Results: Among all the developed polymeric Nanoparticle formulations, optimized formulation (F1) showed Zeta Particle size 269 nm, Encapsulation Efficiency $90.1 \%$. In vitro release studies exhibited $98.78 \%$ of drug release within $3 \mathrm{hr}$ and ex-vivo studies indicate optimize formulation is nonirritant. Differential Scanning Calorimetry and X-Ray Diffraction indicate the optimized formulation show amorphous form. Scanning Electron Microscopy of optimized formulation show cylindrical in shape and their size was in the nanometer range. In terms of particle size, drug loading
\end{abstract}

performance, redispersibility, in vitro release characteristics, sterility and better ocular tolerability, the formulated acyclovir nanoparticles were found to be a suitable and potential natural carrier. Suitable results have been shown in the stability study of acyclovir from nanoparticles. Conclusion: Therefore, the results concluded that Polymeric Nanoparticle could be considered as ocular delivery system for the prolong residence time of precorneal drug and increase penetration of corneal drug.

Key words: Acyclovir, Polymeric nanoparticle, Poly Lactic-Co-Glycolic Acid, Ocular drug delivery, Nanoprecipitation.

\section{Correspondence}

Mr. Harshal D Mahajan

Assistant Professor, Institute Name and Postal Address: DCS's A.R.A. College of Pharmacy, Mumbai-Agra Road Nagaon, Dhule-424005, Maharashtra, INDIA.

Email: h.d.mahajan@gmail.com

DOI: 10.5530/ijpi.2021.1.12

\section{INTRODUCTION}

Ocular drug delivery devices are designed to treat the eye locally, whereas earlier formulations are aimed at achieving systemic circulation and all the drawbacks of traditional treatment types, such as ophthalmic solutions, are supposed to be overcome. Eye discomfort that induces lacrimation due to pharmacokinetic reactions such as pharmacokinetic reactions is the solution issue with conventional dosage types. ${ }^{1}$ The traditional dosage type i.e. eye drops are troublefree to inculcate but suffer from the inherent disadvantage that the mainstream of the drug it contains is dissolved instantly in the tear film as soon as the eye drop solution is instilled into the cul-de-sac and is easily drained away from the precorneal cavity by steady tear flow, a cycle that continues more intensively in inflamed than in than in the lacrimal-nasal drainage normal eyes and normal eyes. ${ }^{2}$

For superior eye bioavailability, a combination of eye drug delivery systems such as inserts and collagen shields and colloidal systems such as liposomes, nanoparticles and nanocapsules have been established and researched. The use of drug delivery systems focused on nanotechnology, such as microemulsions, nanosuspension, nanoparticles, solid lipid nanoparticles, niosomes, dendrimers and liposomes, has helped solve numerous problems of poorly soluble solubility-related drugs. ${ }^{3}$ Ocular efficacy is closely linked to the bioavailability of ophthalmic drugs, which can be enhanced by increasing corneal drug penetration and extending precorneal residence period. The design of polymeric nanoparticles is one of the techniques currently used to improve drug absorption across biological membranes.

It was suggested that the use of mucoadhesive polymers would increase the concentration and time of residence of the associated drug, taking into account the short residence in the ocular cavity of the dosage form. ${ }^{4}$ Based on journalism facts, the three most frequently used polymers in ophthalmic formulations are poly (alkyl cyanoacrylates), polycaprolactone and Poly D, L-lactide. Facts from the literature also confirm that for ophthalmic drug delivery, a sufficient amount particle size and a narrow size range should be needed for each suspended drug, ensuring low discomfort, proper bioavailability and compatibility with ocular tissues. Along with the large variety of mucoadhesive polymers published in the journalism, the Poly D, L-lactide was elected as a choice polymer due to its distinctive uniqueness including logical biodegradability, biocompatibility and increased membrane permeability. ${ }^{5,6}$

Acyclovir is an antiviral drug that has a important and highly selective effect on herpes viruses and is utilized to treat different eye diseases.? The normal use of acyclovir, now commonly available as ointments and drops, is managed by little corneal drug penetration, poor eye bioavailability and systemic exposure to pulse drug entry due to nasal drainage and nasolacrimal duct into the rear eye segments due to the lens-iris diaphragm. As a result, for successful ocular delivery of the drug with enhanced bioavailability the improvement of acyclovir-loaded polymeric nanoparticles was undertaken. The present work was aimed to create and characterize acyclovir-containing biodegradable nanoparticles to optimize precorneal residence time and eye bioavailability. ${ }^{8}$

\section{METHODS}

Acyclovir was procured from Cipla Ltd., Mumbai, India as a gift sample. Polyvinyl alcohol (PVA) M. Wt. 22000 Dialysis tubing cellulose 
membrane which is having molecular weight cut-off 12000-14000 g/ mole was purchased from Sigma Aldrich Pvt Ltd., Mumbai, India. Poly Lactic-Co-Glycolic Acidester having viscosity of $0.16-0.24 \mathrm{dL} / \mathrm{g}$ was purchased from Sigma Aldrich Pvt. Ltd., Mumbai. All other reagents used were of analytical grade.

\section{Preparation of drug loaded nanoparticles using nanoprecipitation technique}

For the preparation of the acyclovir nanoparticles, the nanoprecipitation technique was used. A biodegradable polymer (Poly Lactic-Co-Glycolic Acid) organic solution and an exact amount of $50 \mathrm{mg}$ acyclovir were prepared in $10 \mathrm{~mL}$ acetone. The organic phase was added dropwise into $20 \mathrm{~mL}$ of aqueous solution containing PVA (1\%) and stirred magnetically. After 30 min of stirring, the dispersion volume of nanoparticles was concentrated under reduced pressure to $10 \mathrm{~mL}$ using a vacuum Rota evaporator (KNF, vaccum pumps and system). The aggregates were removed by filtration through a $0.45 \mu \mathrm{m}$ syringe filter. Separation of non-encapsulated drug was performed by ultracentrifugation (Beckman Coulter) at 50,000rpm at $4^{\circ} \mathrm{C}$ for $30 \mathrm{~min}$. The supernatant was discarded and divided nanoparticles were washed twice with distilled water to remove excess surfactant. The washed particles were resuspended and freeze-dried for $48 \mathrm{hr}$ in $5 \mathrm{~mL}$ of water solution containing 5 percent $(\mathrm{w} / \mathrm{v})$ mannitol as a cryoprotectant. The whole experimental carried out in aseptic area. For further investigation the nanoparticles were kept at $2^{\circ} \mathrm{C}$ to $8^{\circ} \mathrm{C}$.

\section{Design of the experiment}

To investigate the effect of various parameters on the physico-chemical properties of the prepared nanoparticles, $3^{2}$ full factorial designs were used as shown in Table 1 and Table 2 . As independent variables, polymer concentration and varying velocities were picked. The particle sizes of the drug's colloid framework and encapsulation capacity have been chosen as the dependent variables.

\section{Characterization of the nanoparticles}

\section{Determination of the particle size}

Photon correlation spectroscopy with Zeta-particle size, Model Nano ZS, was used to determine the size distribution, average particle size and PDI. The isolated nanoparticles were subjected to dilution with distilled water accompanied by calculation. Measurements of the particle size and PDI were carried out at a dispersion angle of $90^{\circ}$ and a temperature of $25^{\circ} \mathrm{C} .^{9}$

\section{Encapsulation efficiency measurements}

Extraction and quantification of encapsulated acyclovir determined the efficacy of the encapsulation of acyclovir in the polymeric nanoparticle. Acyclovir polymeric nanoparticles were added to $25 \mathrm{ml}$ methylene chloride and subjected to $12 \mathrm{~h}$ room temperature shaking to ensure complete particle dissolution. The resulting solution was diluted with appropriate dilution and the concentration of the drug in methylene chloride was determined by Spectrophotometric by measuring UV absorbance at $253 \mathrm{~nm} .^{10}$

$\mathrm{EE}$ of the drug= (amount of encapsulated drug) / (total amount of the drug) X 100

Equation No.1

\section{Redispersibility of nanoparticles}

The chosen formulation was freeze-dried in order to acquire a dry powder for further investigation. Furthermore, the effect of the cryoprotectant on the freeze-drying and dispersibility of the nanosuspension prepared has been revised. At a concentration of 5 times the total solid content of the formulation, Mannitol is used as a cryoprotectant. Two nanosuspension samples were each put in a flask, the quantity of critical mannitol applied to one and shaken to dissolve, the second sample left with cryoprotectants. These flasks were frozen for primary freezing in a deep freezer at $-20^{\circ} \mathrm{C}$ for $12 \mathrm{~h}$. Then the jar was connected to the lyophilizer vacuum adapter. The solvent sublimed under a pressure of $80 \mathrm{mmHg}$ for $48-72 \mathrm{~h}^{11}$

\section{Differential Scanning Calorimetry}

For differential scanning calorimetric measurements, Differential Scanning Calorimeter (DSC DA 60 Shimadzu, Japan) equipped with liquid nitrogen subambient accessory was used. The DSC was performed for the pure acyclovir, the Poly Lactic-Co-Glycolic Acid and the drug loaded nanoparticle formulation. Sample $2 \mathrm{mg}$ were loaded in a flatbottomed aluminum pan and subjected to a heating cycle from 40 to $400^{\circ} \mathrm{C}$ with a heating rate of $10^{\circ} \mathrm{C} / \mathrm{min}$. To control the heating and cooling rate,a stream of nitrogen gas was used. Purified indium as the reference material was used for the calibration of the temperature and energy scales of the instrument. ${ }^{12}$

\section{X-ray diffraction}

X-ray diffraction analysis was used in nanoparticle formulation to detect the drug's crystallinity, which was conducted with a copper target and nickel filter using a Philips PW 3710 X-ray diffractometer (XRD). Powders were installed with glass bottoms on aluminium stages and smoothed down to a level foundation. Each sample's XRD pattern was calculated from 10 to 50 degrees 2-theta using a phase increase of 0.1 degrees 2 -theta and a dwell time of 1 second at each step. ${ }^{13}$

\section{Scanning Electron Microscopy}

Scanning electron microscopy (JEOL Model JSM-6390LV) has been used to describe the surface morphology of nanoparticles. Using doublesided, platinum-coated sticking tape, the nanoparticles were directly placed on the SEM stub and screened in a high vacuum chamber with a directed electron beam. Secondary electrons were detected, released from the samples and the picture formed. ${ }^{14}$

\section{Swelling Index}

The exactly weighted nanoparticles were put in the incubator in a glass vial containing a $10 \mathrm{~mL} \mathrm{pH} 7.4$ phosphate buffers at $37 \pm 0.5^{\circ} \mathrm{C}$ and were periodically stirred. Using philtre paper, the nanoparticles were periodically extracted by blot and the change in particulate weight was determined before balancing. The weight was registered in triplicate after duration of $3 \mathrm{~h}$ and the swelling ratio (SR) was calculated with the formula (Eq. 2). ${ }^{15}$

Swelling index $(\%)=\mathrm{W} 1-\mathrm{W} 2 \mathrm{X} 100 / \mathrm{W} 1$

Equation No. 2

Where,

$\mathrm{W} 1=$ Weight of nanoparticles after swelling

W2 = Initial weight of nanoparticles

\section{Sterility Testing}

Sterility tests have been carried out to guarantee the final product's sterility. The direct inoculation approach was chosen to perform sterility checking since it is administered by parenteral path. During this process, the individual sample quantity under test was extracted aseptically from the containers and transferred separately to the fluid thioglycollate medium $(20 \mathrm{~mL})$ and the soybean casein digest medium $(20 \mathrm{~mL})$. In the case of fluid thioglycolate medium and $20^{\circ} \mathrm{C}-25^{\circ} \mathrm{C}$ in the case of SoybeanCasein digest medium, the mixture of nanoparticles with the medium was incubated at $30^{\circ} \mathrm{C}-35^{\circ} \mathrm{C}$ for not less than 14 days. Development was observed of any micro-organisms in the medium. ${ }^{16}$ 


\section{In vitro Release Studies}

A membrane diffusion technique was used to conduct a release analysis of the nanoparticle formulation for the release of acyclovir from polymeric nanoparticles. Using the dialysis membrane as a semipermeable membrane, in vitro diffusion cells were developed. A dialysis membrane of 12,000-14,000 Molecular weight cut-off was used to maintain the nanoparticles as well as to enable the free drug to diffuse freely into the release media. Formulated nanoparticles equivalent to 1 $\mathrm{mg}$ of the drug were dispersed in $1 \mathrm{~mL}$ of isotonic phosphate-buffered saline at $\mathrm{pH}$ 7.4. The nanoparticle dispersions were packed in dialysis membrane secured with two clamps at each end. The dialysis bag was submerged in tightly-capped glass vials $(7 \times 2.8 \mathrm{~cm})$ containing $10 \mathrm{~mL}$ of 0.5 percent $(\mathrm{w} / \mathrm{v})$ sodium lauryl sulphate solution in distilled water in order to preserve sink conditions. The release test was conducted by putting the glass vials in a thermostatically operated shaking water bath with a constant shaking rate of $100 \mathrm{rpm}$, set to $37 \pm 0.5^{\circ} \mathrm{C}$. The entire release medium was discontinued and replaced at fixed time points with a new release medium.By UV spectrophotometry, the concentration of the drug released was measured. Almost all studies were performed in triplicates. ${ }^{17}$

\section{Chorioallantoic Membrane Test and Irritation Score Calculation}

A chorioallantoic membrane (CAM) test was carried out as a mucousmembrane irritation test for the Draize eye irritation test. The test was carried out using commercially available mycoplasma-free fertilized white chicken eggs. The hen's eggs were put in the incubator trays with the wide ends up for CAM testing; the trays were placed in the incubator, which rotates automatically and was held at an optimal temperature of $37.5 \pm 0.5^{\circ} \mathrm{C}$. On day 5 of incubation and everyday afterwards the eggs were candled, eliminating nonviable embryos. On day 10 of incubation the egg shell was scratched around the air cell by a dentist's rotary

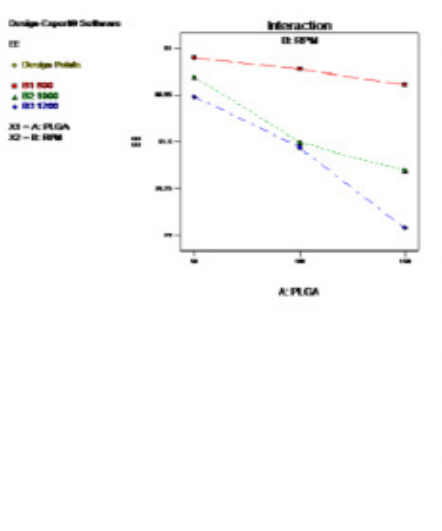

A)

Design-Expert ${ }^{\oplus}$ Software

Interaction

Particle size

275

Design Points

B1 800

B2 1000

B3 1200

$\mathrm{X} 1=\mathrm{A}: \mathrm{PLGA} 239$

$\mathrm{X} 2=\mathrm{B}: \mathrm{RPM}$

Figure 1: Surface plots showing the effect of (A) Encapsulation Efficiency (B) Particle Size. saw and then pared off. After carefully removal of the internal egg membranes the vascular CAM was revealed. The test sample was applied to the CAM surface at a volume of $0.2 \mathrm{ml}$. A set of four eggs were used; two vehicle-only eggs acted as controls. For irritant effects (hyperaemia, haemorrhages, coagulation), CAM, blood vessels, including the capillary system and albumen were tested and rated at 0.5, 2 and $5 \mathrm{~min}$ after treatment following application of the test drug. Numerical timedependent scores for hyperaemia, haemorrhages and coagulation (Table 3) have been summarized to provide a single numerical value indicating the discomfort potential of the test product on a scale with a maximum value of 21. An evaluation by a classification scheme similar to the Draize categories (Table 4) allows for the mean value of four measures. ${ }^{18}$

\section{Stability Studies}

Stability tests were performed out in the stability chamber (Thermo lab) for 6 months on selected formulations at $30 \pm 2^{\circ} \mathrm{C}$. The optimized formulation stored in the sealed in glass bottle. Drug content, particle size and redispersibility studies were carried out after 6 months. ${ }^{19}$

\section{RESULTS}

\section{Experimental Design}

Two factors (polymer concentration and homogenizer speed) were calculated based on the experimental and above studies crucial factor for particle size and encapsulation efficiency of prepared polymeric nanoparticles as shown in Figure $1 .^{20}$

\section{Preparation of drug loaded nanoparticles}

Using the rapid and simple nanoprecipitation technique acyclovir loaded Poly Lactic-co-glycolic acid Polymeric nanoparticles were successfully prepared. The formation of nanoparticles is one phase and an instant procedure. As the non-solvent is applied to the polymer solution, rapid desolation occurs accompanied by nanoprecipitation. As soon as the polymer containing solvent has diffused into the dispersing medium, the polymer precipitates, requiring immediate drug treatment.

\section{Characterization of nanoparticles Determination of particle size}

The mean particle size of the formulae of prepared nanoparticles is shown in Table 5. The size of the particles ranges between 203.6 and $274.9 \mathrm{~nm}$. For all formulations of nanoparticles, the particle size distribution indicated by the polydispersity index (PDI) was calculated. Average PDI values vary from 0.555 to 1.0 .0 .

\section{Encapsulation Efficiency}

Nanoparticle encapsulation efficiency \% ranged between 72.7 to $90.1 \%$. All tested variables have a significant effect on EE\%.

\section{Redispersibility Test}

It was found that the dispersibility was improved by using mannitol as cryoprotectants and products in both media were spontaneously dispersed through primary nanosuspension within $1-3 \mathrm{~min}(0.1 \mathrm{~N} \mathrm{HCl}$ and phosphate buffer $\mathrm{pH}$ 6.8).

\section{Differential Scanning Calorimetry}

To verify the existence in the physical interaction between acyclovir and excipients each sample was analysed by differential scanning calorimetry (DSC). DSC thermogram of acyclovir, Poly Lactic-Co-Glycolic Acid and lyophilized drug-loaded nanoparticles is shown in Figure 2. The characteristic endothermic peaks at $254^{\circ} \mathrm{C}$ of the drug disappeared in the drug loaded nanoparticles thermogram. ${ }^{21}$ 


\section{X-Ray Diffraction}

To determine the crystallinity of the acyclovir in the nanoparticles, Powder $\mathrm{X}$ ray diffraction was used. The powder $\mathrm{X}$ ray diffraction patterns of optimized formulation (F1) are shown in Figure 3.

\section{Scanning Electron Microscopy (SEM)}

The morphology of drug loaded nanoparticles (F1) was accessed using SEM and is shown in Figure 4.

\section{Swelling Index}

Exploration increase in swelling index from $57 \%$ to $80 \%$ as polymer concentration is increase from $50 \mathrm{mg}$ to $150 \mathrm{mg}$ as shown in Figure 5.

\section{Sterility Testing}

Formulations have been incubated at $30^{\circ} \mathrm{C}$ to $35^{\circ} \mathrm{C}$ for no less than 14 days in the case of a fluid thioglycolate medium and at $20^{\circ} \mathrm{C}$ to $25^{\circ} \mathrm{C}$ in the case of a soybean casein digest medium, showing that the formulation passes the sterility examination.

\section{In vitro drug release from nanoparticles}

The drug formulae prepared with nanoprecipitation technique were subjected to in vitro release study. Dialysis technique was use for the evaluation of the amount of acyclovir released from nanoparticles. The release profiles of acyclovir are shown in Figure 6. The in vitro release studies of optimized polymeric nanoparticles exhibited $98.78 \%$ of drug release within $3 \mathrm{hr}$.

\section{Chorioallantoic Membrane Test and Irritation Score Calculation}

It was found that the formulation is non-irritant as score value for Hyperemia, Hemorrhage and coagulation is zero, as compared to phosphate buffer solution $\mathrm{pH} 7.4$ should coagulation after five minutes (Score value 1.4) which is slightly irritant as shown in Figure 7.

Table 1: Experimental plan of the $\mathbf{3}^{2}$ factorial designs.

\begin{tabular}{cccc}
\hline Factor & \multicolumn{3}{c}{ Level } \\
\cline { 2 - 4 } & ++ & + & - \\
\hline Speed of Homogenizer & 1200 & 1000 & 800 \\
$\begin{array}{c}\text { Polymer Concentration } \\
(\mathrm{mg})\end{array}$ & 150 & 100 & 50 \\
\hline
\end{tabular}

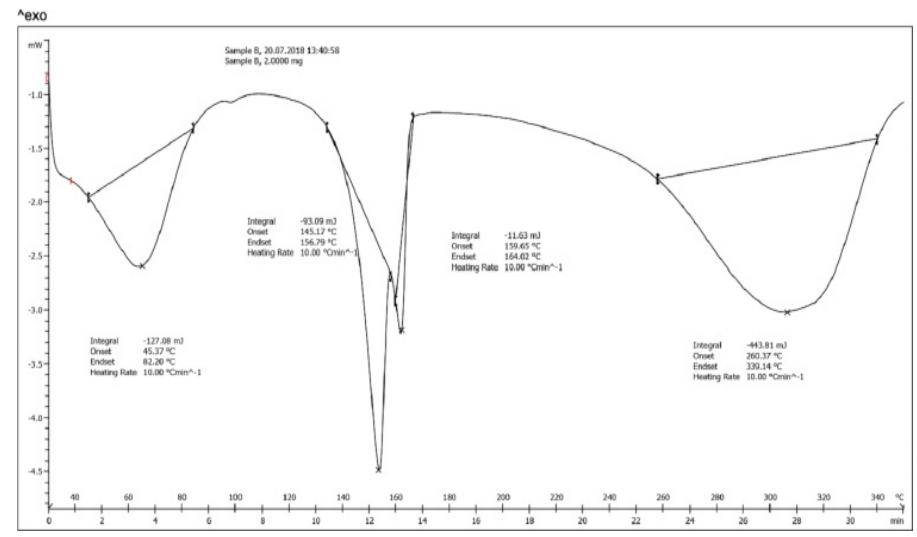

Figure 2: DSC thermogram of the optimized nanoparticles formulation (F1).

\section{Stability Studies}

Stability studies were conductedon optimized formulation at $30 \pm 2{ }^{\circ} \mathrm{C}$ in stability chamber (Thermolab) for 6 month as shown in Table 6 . The optimized formulation stored in the sealed in aluminum foil. Drug content, particle size and redispersibility studies were carried out after 6 months.

\section{DISCUSSION}

The experimental design shows that the concentration of polymer has been shown to have a significant effect on the efficacy of trapping as optimum concentration of polymers. The homogenizer speed also has an effect optimization formulation. Nanoprecipitation technique also generates a carrier size within the range of nanometers and uses low toxic potential ingredients that are crucial for ocular pathways. When considering irritation and comfort, the particle size is significant factor in the development of an ocular drug delivery system. The effect of different variables of formulation, namely concentration and speed of polymers, had a major impact on particle size. Concerning the effect of polymer concentration the results shows that the particle sizes obtained using lower concentration of polymer were significantly smaller than those obtained using higher concentration of polymers. It has also shown that particle size increases with increase in the polymer concentration. Different speed also affects the particle size. Particle sizes obtained by using low speed were significantly larger than the particle sizes obtained

\begin{tabular}{ccc}
$\begin{array}{c}\text { Table 2: Composition of Drug /Polymer Concentration and speed of } \\
\text { different formulation of ophthalmic polymeric nanoparticles. }\end{array}$ \\
\hline $\begin{array}{c}\text { Formula } \\
\text { Number }\end{array}$ & $\begin{array}{c}\text { Drug : Polymer } \\
\text { Concentration }\end{array}$ & Speed \\
\hline F1 & $1: 1$ & 800 \\
F2 & $1: 2$ & 800 \\
F3 & $1: 3$ & 800 \\
F1 & $1: 1$ & 1000 \\
F5 & $1: 2$ & 1000 \\
F6 & $1: 3$ & 1000 \\
F7 & $1: 1$ & 1200 \\
F8 & $1: 2$ & 1200 \\
F9 & $1: 3$ & 1200 \\
\hline
\end{tabular}

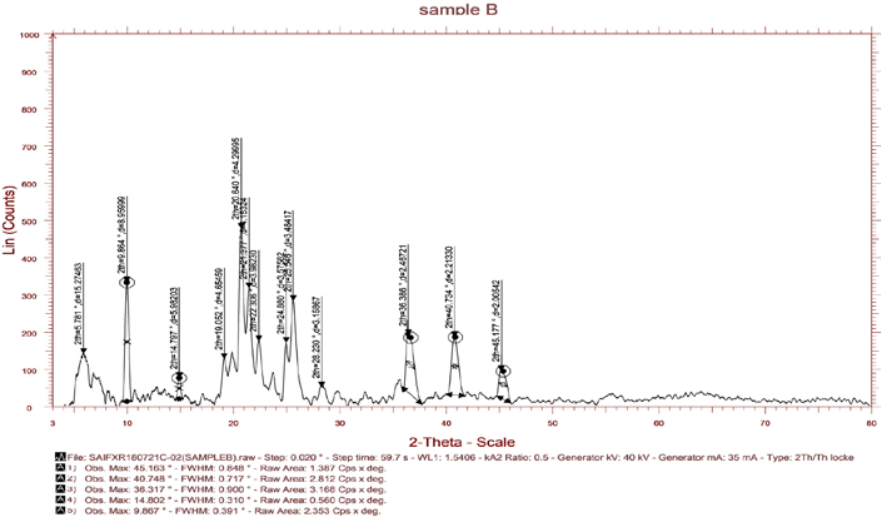

Figure 3: XRD of the optimized nanoparticles formulation (F1). 
by using high speed. So the speed which gave the smaller particle size was speed used for F1. The narrow distribution represented by the small PDI values denotes particle size uniformity in the nanoparticle formulae. Encapsulation Efficiency studies suggested that the increase in polymer content resulted in a decrease in the EE percentage of formulae for nanoparticles. This finding can be due to the rising viscosity of the organic process when the polymer content increases. The redispersibility study demonstrated that the wetting of the hydrophobic drug will increase mannitol in the products and accelerate the penetration of water into the products. On the other hand, as

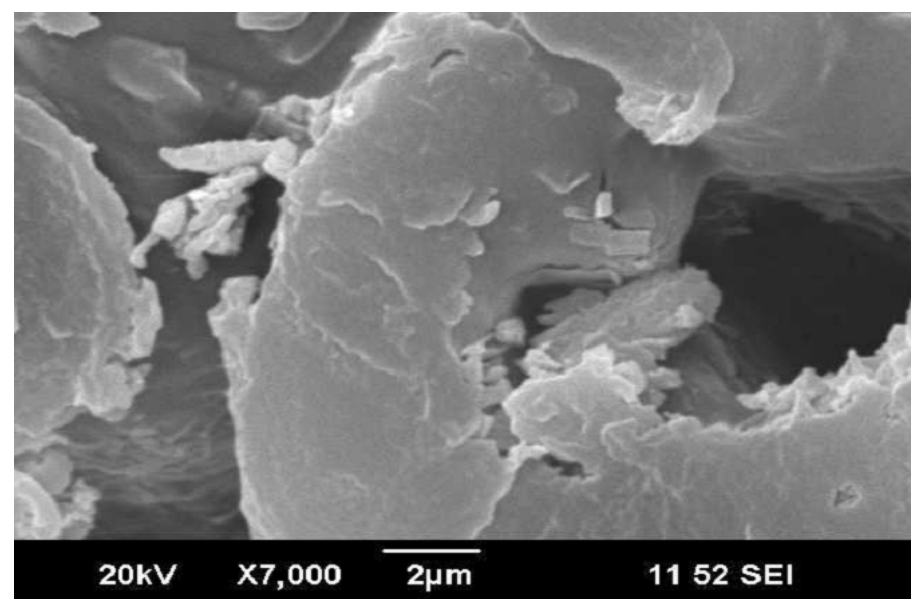

Figure 4: SEM of the optimized nanoparticles formulation (F1).

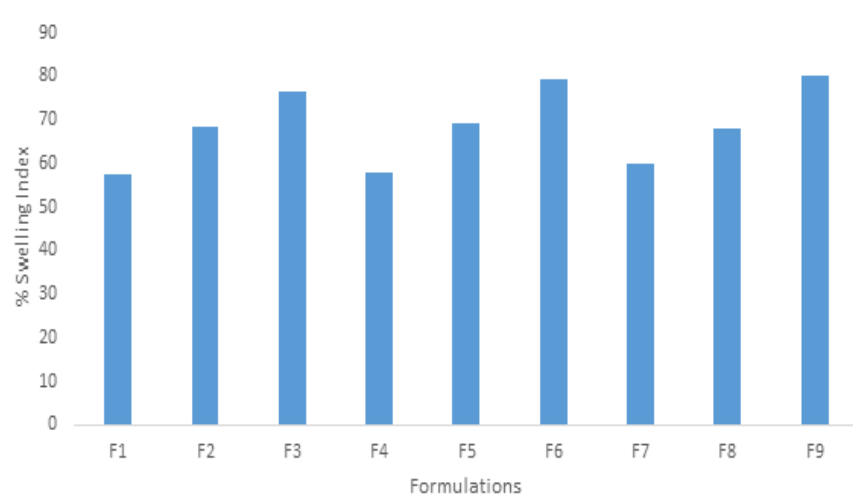

Figure 5: Percentage of Swelling Index of Nanoparticles Formulation.

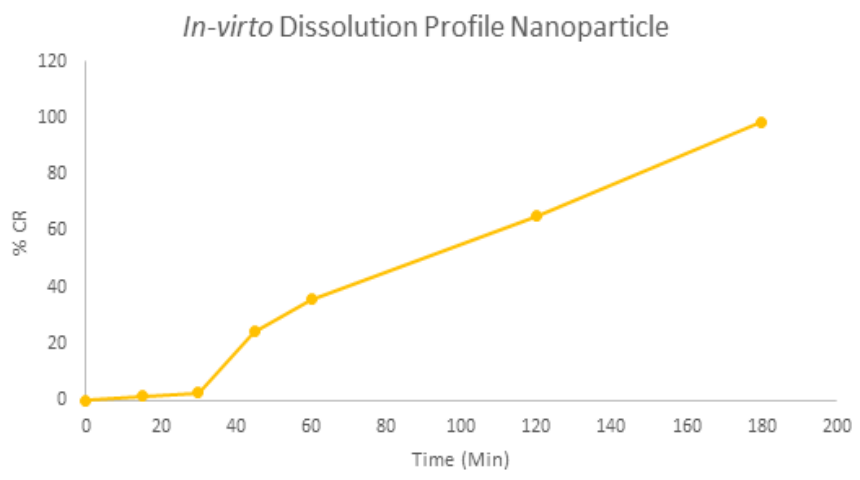

Figure 6: In vitro release study of the Acyclovir nanoparticles formulation.
Table 3: Scoring scheme for irritation testing with the hen's egg chorioallantoic membrane.

\begin{tabular}{cccc}
\hline & \multicolumn{3}{c}{ Score (Time in Second) } \\
\cline { 2 - 4 } & 0.5 & 2 & 5 \\
\hline Hyperemia & 5 & 3 & 1 \\
Hemorrhage & 7 & 5 & 3 \\
Coagulation & 9 & 7 & 5 \\
\hline
\end{tabular}

Table 4: Classification of cumulative scores in the chorioallantoic membrane test.

\begin{tabular}{cc}
\hline Cumulative Irritation & Score assessment \\
\hline $0-0.9$ & Practically none \\
$1-4.9$ & Slight \\
$5-8.9$ & Moderate \\
$9-21$ & Strong \\
\hline
\end{tabular}

Table 5: Particle Size and Encapsulation Efficiency with different formulation.

\begin{tabular}{ccc}
\hline Formulations & Particle Size $(\mathrm{nm})$ & $\begin{array}{c}\text { Encapsulation } \\
\text { Efficiency (\%) }\end{array}$ \\
\hline F1 & 269 & 90.1 \\
F2 & 271.1 & 88.9 \\
F3 & 274.9 & 87.3 \\
F1 & 235.6 & 88.1 \\
F5 & 240.1 & 81.4 \\
F6 & 247.7 & 78.6 \\
F7 & 203.6 & 86.1 \\
F8 & 210.6 & 80.9 \\
F9 & 219.9 & 72.7 \\
\hline
\end{tabular}

Table 6: Stability of acyclovir nanoparticles during storage (F1).

\begin{tabular}{ccccc}
\hline Parameter & 0 Day & 1 Month & 3 Month & 6 Month \\
\hline Drug Content & 90.1 & 89.9 & 88.7 & 87.1 \\
Particle Size & 269.0 & 270.1 & 271.5 & 272.7 \\
\hline
\end{tabular}

predicted from their agglomerated structure, the products without cryoprotectants could not be well dispersed and converted into the original nanosuspension within $15 \mathrm{~min}$. DSC studies revealed that acyclovir was entrapped in an amorphous or molecular dispersion state in the polymer matrix. The results of XRD indicate that the drug was present in the nanoparticles in an amorphous state, confirming DSC results. The morphology of drug loaded polymeric nanoparticles using Scanning Electron Microscopy indicates that the nanoparticles were cylindrical in shape and their size was in the nanometer range with smooth surface essential for ocular drug delivery. Swelling index study indicates that being Poly Lactic-Co-Glycolic Acid as hydrophilic polymer uptake excessive amount of water responsible for swelling for polymeric nanoparticles. When formulations were subjected to sterility testing, 


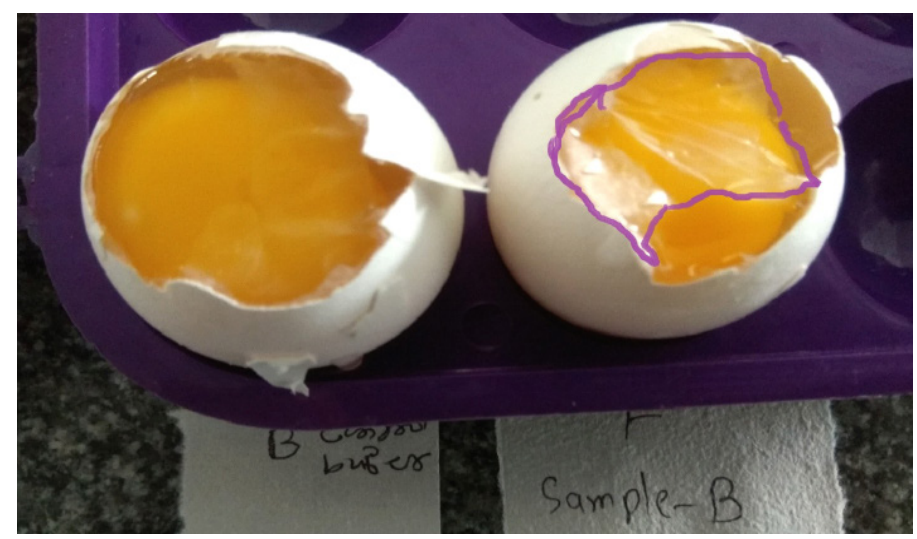

Figure 7: CAM Test of the optimized nanoparticles formulation (F1).

there was no evidence of microbial growth detected. The rapid dissolution mechanism therefore indicates that, because of the hydrophilic nature of PLGA, the release medium penetrates into the particles and dissolves the trapped acyclovir. The release profile of the drug from the nanoparticles showed fitting with Higuchi kinetics. The irritation score of HET-CAM test showed that the developed cationic nanoparticle is essentially nonirritating and possess good ocular tolerability. Stability study suggests that the optimized polymeric nanoparticle formulation was found to be stable over period of 6 months.

\section{CONCLUSION}

Acyclovir was successfully prepared within biodegradable nanoparticle using nanoprecipitation technique. The finest formulation was obtained by using $3^{2}$ factorial designs. The drug-polymer ratio and speed had a noteworthy effect on the particle size and encapsulation efficiency of the nanoparticle. In terms of particle size, drug loading capability, redispersibility, in vitro release characteristics, sterility and better ocular tolerability, the formulated acyclovir nanoparticles were found to be an effective and latent natural carrier. Suitable findings have been shown in the stability analysis of acyclovir from nanoparticles.

\section{ACKNOWLEDGEMENT}

The authors would like to thank DCS's A. R. A. College of Pharmacy, Nagaon, Dhule for providing necessary facilities for pursuing the work.

\section{CONFLICT OF INTEREST}

The authors declare no conflict of interest.

\section{ABBREVIATIONS}

PLGA: (Poly-Lactic-Acid-Co-Glycolic-Acid); PDI: Polydispersity Index; DSC: Differential Scanning Calorimetry; XRD: X-Ray Diffraction; SR:
Swelling Ratio; CAM: Chorioallantoic Membrane Test; SEM: Scanning Electron Microscopy.

\section{REFERENCES}

1. Lang JC. Ocular drug delivery conventional ocular formulation. Advanced Drug Delivery Review. 1995;16(1):39-43.

2. Basavaraj K, Nanjawade MFV, Manjappa AS. In situ forming hydrogels for sustained ophthalmic drug delivery. J Cont Rel. 2007;122(2):119-34.

3. Sahoo KS, Fahima SAD, Kumar K. Nanotechnology in ocular drug delivery. D Deli Tod. 2008;13(3-4):144-51.

4. Weidener J. Mucoadhesive ocular inserts as an improved delivery vehicle for ophthalmic indications. D Disc Tod. 2003;8:906-7.

5. Himanshu G, Mohammed A, Roop KK, Asgar A, Aseem B, Gaurav M, et al. Sparfloxacin-loaded PLGA nanoparticles for sustained ocular drug delivery. Nano NBM. 2010;6(2):324-33.

6. Azza AM, El-Feky GS, Rabab KGEAA. Chitosan/sulfobutylether-B-Cyclodextrin Nanoparticles as a Potential Approach for Ocular Drug Delivery. Int J Pharm. 2011;413:229-36.

7. Rajendran NN, Natrajan R, Siva KR, Selvaraj S. Acyclovir-loaded chitosan nanoparticles for ocular delivery. Asian Jo of Phar. 2010;220-6.

8. Lee VHL, Robinson JR. Topical ocular drug delivery: Recent developments and future challenges. J of Ocu Pharmacol.1986;2(1):67-108.

9. Gupta P, Pandit J, Ajay P, Swaroop P, Gupta S. Pharmaceutical Nanotechnology Novel Nanoemulsion-High Energy Emulsification Preparation. Evaluation and Application T Pharma Res. 2010;3(3):117-38.

10. Abdel HSA, Salah S. Development of nanoparticulate formulations for ocular delivery of prednisolone acetate: Preparation and characterization. Drug Del Sci Tech. 2014;24(2);159-65.

11. Viswanath V, Narasimha B, Rao K, Gnana P, Rahath FS, Krishnaveni G. Formulation and evaluation of Terbulatine sulphate loaded inhalation nanoparticles for pulmonary drug delivery system. Int J Pharm Sci Rev Res. 2017;42(1):256-60.

12. Khyati K, Kumar $P$, Jalpa SP, Moinnudin MS. Formulation And Evaluation of Nanosuspension to Improve Solubility And Dissolution of Diacerein. Int J Pharma Sci Res. 2017;8(4):1643-53.

13. Sankar C, Muthukumar S, Arulkumaran GS, Shalini RSNV. Design and Development of Bendamustine loaded Chitosan Nanoparticle in-vitro Cytotoxicity. Int J Pharma Sci Res. 2020;11(2);755-64.

14. Shiva T, Shahla M. Preparation and characterization of Novel, Mucoadhesive Ofloxacin nanoparticles for ocular drug delivery. B J Pharm Sci. 2019;55:1-12.

15. Rekha KJS, Vinay S. Development and Characterization of Nanoparticles for the Delivery of Gemcitabine Hydrochloride Hindawi Publishing Corporation. Sci W J. 2014;1-6.

16. Higuchi T. Rate of release of medicaments from ointment bases containing drugs in suspension. J Pharma Sci. 1961;50(10):874-5.

17. Lupepke NP. Hen's Egg Chorioallantoic Membrane Test for Irritation Potential. Fd Chem Toxk. 1985;23(2):287-91.

18. Drug Stability: Principle and Practices, third edition, revised and expanded edited by Jens T. Carstensen and C.T. Rhodes, Drug and Pharmaceutical Science, Marcel Dekker New Work. 2002;107.

19. BibaswanM, JagannathS. Enhanced bioavailability of cinnarizinenanosuspensions by particle size engineering: Optimization and physicochemical investigations. Mater Sci Eng C. 2016;63(1):62-29.

20. Payal P, Pooja W, Mahajan HLZ. Aripiprazole loaded polymeric micelles: Fabrication, optimization and evaluation using response surface method. Rec Pat D Deli Form. 2018;12(1):1-7.

21. Gupta P, Chawala G, Bansal AK. Physical stability advantages from amorphous celecoxib: the role of thermodynamic quantities and molecular mobility. Mol Pharm. 2014;1(6):406-13. 